\title{
Light in dielectric media and scalar fields in a de Sitter spacetime
}

\author{
I. A. Pedrosa ${ }^{\mathrm{a}}$, B. F. Ramos ${ }^{\mathrm{b}}$, K. Bakke \\ Departamento de Física, CCEN, Universidade Federal da Paraíba, Caixa Postal 5008, João Pessoa, PB 58059-900, Brazil
}

Received: 6 May 2021 / Accepted: 26 July 2021 / Published online: 6 August 2021

(C) The Author(s) 2021

\begin{abstract}
In the present work we discuss the behavior of light in a linear dielectric medium with a time-varying electric permittivity that increases exponentially at a constant rate and of a scalar field in a de Sitter spacetime, in both the classical and quantum contexts. Notably, we find that the behavior of these two systems are identical and can be described by similar Hamiltonians. By using the Lewis-Riesenfeld invariant method together with Fock states we solve the timedependent Schrödinger equation for this problem and use its solutions to construct coherent states for the scalar field. Finally, we employ both the Fock and coherent states to evaluate some important properties of the quantized scalar field, such as expectation values of the amplitude and momentum of each mode their variances and the respective uncertainty principle.
\end{abstract}

\section{Introduction}

In the past few years, the investigation of the propagation of light in material media, in both the classical and quantum contexts, has received a lot of attention of physicists. This great interest is due to the fact that the solution of this problem is very important for the development of our understanding of nature. In particular, a variety of work on this propagation in a time-dependent dielectric medium has been published in the literature recently [1-12].

Another subject that has attracted the attention of theoretical physicists in recent years is the classical and quantum propagation of scalar fields in curved spacetimes. In particular, the investigation of scalar fields propagating in a de Sitter spacetime has been recently reported in the physical literature [13-21]. On the other hand, it is needless to say that the study of physical systems that present similar behavior

\footnotetext{
a e-mail: iapedrosa@ fisica.ufpb.br (corresponding author)

b e-mail: bugley_15@hotmail.com

c e-mail:kbakke@ fisica.ufpb.br
}

and are described in the same mathematical framework is of special interest in the context of theoretical physics, since the elucidation of similarities of systems studied by different areas of physics frequently allows the knowledge of each branch to help the understanding of the other. Here it is worth remarking that light propagating in a time-dependent dielectric medium and the propagation of scalar fields in a de Sitter spacetime belong to different areas of physics.

In the present work, stimulated by these trends in this research field, we discuss the propagation of light in a dielectric medium with a time-varying permittivity increasing exponentially and scalar fields propagating in a de Sitter spacetime. We demonstrate that the mathematical framework to study this propagation is identical, both classically and quantum mechanically, and can be described by similar Hamiltonians. Further, by using the dynamical invariant method and Fock states we solve the time-dependent Schrödinger equation for the Hamiltonian associated with the scalar field and write its solutions in terms of the nonlinear Milne-Pinney equation. We also use these solutions to construct coherent states for the quantized scalar field. Finally, we evaluate various physical properties for the quantized scalar field, such as the expectation values of the amplitude and momentum for each mode, their quantum variances and the corresponding uncertainty principle.

Our plan for the present paper is as follows. In Sect. 2, by making use of the Coulomb gauge we discuss the classical behavior of the propagation of light in the dielectric medium with a time-dependent permittivity that increases exponentially. In Sect. 3, we investigate the classical behavior of scalar fields in a de Sitter spacetime. In Sect. 4, with the aid of the invariant method and Fock states we solve the timedependent Schrödinger equation associated with the Hamiltonian that describes the scalar field and employ its solutions to derive some physical properties of the quantized scalar field. In Sect. 5, we construct coherent states for the quantized scalar field in a de Sitter spacetime and calculate the expectation values of the amplitude and momentum of each 
mode, their quantum variances and the corresponding uncertainty principle. We conclude our work in Sect. 6 with a short summary.

\section{Classical light in a time-dependent dielectric medium}

In this section our objective is to discuss the classical behavior of the propagation of light in a time-dependent linear dielectric medium. As it is well-known, this propagation is governed by Maxwell's equations and in homogeneous linear media the fields satisfy the relations $\vec{D}=\varepsilon(t) \vec{E}$ and $\vec{B}=\mu_{0} \vec{H}$. Here, $\varepsilon(t)$ and $\mu_{0}$ are, respectively, the electric permittivity and magnetic permeability which represent the properties of the dielectric medium. We consider $\varepsilon$ to be time-dependent because we want to explore the relation of this time dependence with the de Sitter spacetime. Particularly, we consider a time-varying permittivity increasing exponentially in the form [13]

$\varepsilon(t)=\varepsilon_{0} e^{\eta t}$,

where $\eta$ is a positive constant and $\varepsilon(0)=\varepsilon_{0}$. Strictly speaking, the electric permittivity and magnetic permeability are complex but in this work we assume that they are real. The time-dependent model for the permittivity given in Eq. (1) is convenient because it allows (as we will see later) one to establish a direct connection with the de Sitter spacetime. Furthermore, this model has been used in the literature to study many physical problems in curved spacetimes $[13,14,16-19]$. In particular, it has been used to study ion trap simulations in a de Sitter spacetime [22,23]. Also, it is worth to mentioning that Maxwell's equations in the presence of a gravitational field, the gravitational field plays the role of a dielectric medium with electric permittivity $\varepsilon$ and magnetic permeability $\mu$ (see Ref. [24]).

Now, since the Maxwell equations are gauge invariant, we are free to choose the most appropriate gauge for our problem. For convenience and simplicity, we choose to work in the Coulomb gauge $[1,4,7]$. In this gauge the divergence of the vector potential $\vec{A}$ is zero and the scalar potential is null in the absence of sources. Besides, in the Coulomb gauge the vector potential is purely transverse. Thus, using Maxwell's equations it is easy to verify that it satisfies the damped wave equation $[4,13,14]$

$\nabla^{2} \vec{A}-\mu_{0} \dot{\varepsilon} \frac{\partial \vec{A}}{\partial t}-\mu_{0} \varepsilon \frac{\partial^{2} \vec{A}}{\partial t^{2}}=0$

where the dot indicates a time-derivative.

Next, we move our attention to the solution of Eq. (2). By using the familiar procedure of separation of variables, we write the vector potential $\vec{A}$ in terms of the mode $\vec{u}_{l}(\vec{r})$ and amplitude $q_{l}(t)$ functions of each cavity mode as $[1,4]$

$\vec{A}(\vec{r}, t)=\sum_{l} \vec{u}_{l}(\vec{r}) q_{l}(t)$

If we substitute $\vec{A}(\vec{r}, t)$ into the damped wave equation (2) we obtain

$$
\begin{array}{r}
\nabla^{2} \vec{u}_{l}(\vec{r})+\frac{\omega_{l}^{2}}{c_{0}^{2}} \vec{u}_{l}(\vec{r})=0, \\
\frac{\partial^{2} q_{l}}{\partial t^{2}}+\frac{\dot{\varepsilon}}{\varepsilon} \frac{\partial q_{l}}{\partial t}+\Omega_{l}^{2}(t) q_{l}=0,
\end{array}
$$

where $\omega_{l}$ is a separation constant that stands for the natural frequency, $c_{0}=1 /\left(\mu_{0} \varepsilon_{0}\right)^{1 / 2}$ is the velocity of light at $t=0$ and $\Omega_{l}(t)$ is the time-dependent modified frequency defined as

$\Omega_{l}(t)=\frac{c(t) \omega_{l}}{c_{0}}$

with $c(t)=1 / \sqrt{\mu_{0} \varepsilon(t)}$ being the velocity of light in the time-dependent medium. Note that $\Omega_{l}(0)=\omega_{l}$. Now, it is easy to verify that Eq. (5) can be obtained from the classical Hamiltonian

$H_{l}(t)=\frac{p_{l}^{2}}{2 \varepsilon(t)}+\frac{1}{2} \varepsilon(t) \Omega_{l}^{2}(t) q_{l}^{2}$

where $q_{l}$ and $p_{l}$ are conjugate dynamical variables. Now, by making use of expressions (1) and (6), Eq. (5) is transformed into

$\ddot{q}_{l}+\eta \dot{q}_{l}+e^{-\eta t} w_{l}^{2} q_{l}=0$.

The solution of this equation is $[13,14]$

$q_{l}(t)=e^{-\eta t / 2}\left[A J_{1}\left(\frac{2 w_{l}}{\eta} e^{-\eta t / 2}\right)+B Y_{1}\left(\frac{2 w_{l}}{\eta} e^{-\eta t / 2}\right)\right]$,

where $J_{1}$ and $Y_{1}$ are Bessel functions of first and second kind, respectively, and $A$ and $B$ are constants.

Let us now consider the solutions of Eq. (4). Considering that light is contained in a certain cubic volume $V$ of side $L$ of nonrefracting media, the set of vector mode functions $\vec{u}_{l}(\vec{r})$ are required to satisfy the transversality condition $\nabla \cdot \vec{u}_{l}(\vec{r})=0$ and to form a complete orthonormal set. Furthermore, assuming periodic boundary conditions on the surface, the plane wave mode functions appropriate to a cubical volume of side $L$ may be written as $[1,4,7]$

$\vec{u}_{l v}(\vec{r})=L^{-3 / 2} e^{ \pm i \vec{k}_{l} \cdot \vec{r}} \hat{e}_{l v}$, 
where $L=V^{1 / 3}$ is the size of the cube, $\left|\vec{k}_{l}\right|=\omega_{l} / c_{0}$ is the wave vector, and $\hat{e}_{l v}$ are unit vectors in the directions of polarization $(v=1,2)$, which must be perpendicular to the wave vector because of the transversality condition. Hence, by using Eq. (10) we can write the vector potential $\vec{A}(\vec{r}, t)$ (see Eq. (3)) as

$\vec{A}(\vec{r}, t)=\frac{1}{L^{3 / 2}} \sum_{l} \sum_{\nu=1,2} \hat{e}_{l \nu} e^{ \pm i \vec{k}_{l} \cdot \vec{r}} q_{l}(t)$,

with $q_{l}(t)$ given by Eq. (9). Therefore, the above result give us a classical description of the propagation of light in a dielectric linear medium with a time-varying permittivity increasing exponentially since in the Coulomb gauge the electric and magnetic fields are given by $\vec{E}=-\partial \vec{A} / \partial t$ and $\vec{B}=\nabla \times \vec{A}$. Moreover, from Eqs. (9) and (11) we see that the vector potential decay in proportion to $\exp (-\eta t / 2)$. This attenuation in the potential vector is due to time dependence of the electric permittivity (see Eq. (5)) [13]. At this point, it is worth mentioning that, for $\varepsilon$ constant, the Hamiltonian (7) reduces to that of the standard harmonic oscillator with the permittivity $\varepsilon$ playing the role of the mass of the mechanical oscillator. Consequently, all of our previous results coincide with those of the propagation of light in empty cavities.

\section{Classical scalar field in a de Sitter spacetime}

In this section we want to investigate the behavior of classical scalar fields in a de Sitter spacetime. In order to do this, we consider a massive scalar field in the de Sitter background. The de Sitter spacetime has the line element given by $[16,18-$ 20]

$\mathrm{d} s^{2}=-\mathrm{d} t^{2}+e^{2 H_{0} t} \mathrm{~d} x^{2}$

where $H_{0}$ is the time-independent Hubble constant. The Lagrangian density for the real massive scalar field $\Phi(\vec{x}, t)$ is given by $[16,18-20]$

$\mathcal{L}=-\frac{1}{2} g^{\mu \nu} \partial_{\mu} \Phi \partial_{\nu} \Phi-\frac{1}{2} m^{2} \Phi^{2}$,

where $m$ is the mass of the field quanta. Then we can decompose the scalar field on a complete basis of mode $\phi_{k}(t)$ and amplitude $v_{k}(\vec{x})$ functions as $[16,18-21]$

$\Phi(\vec{x}, t)=\sum_{k} v_{k}(\vec{x}) \phi_{k}(t)$

where

$v_{k}(\vec{x})=\frac{1}{\sqrt{V}} e^{i \vec{k} \cdot \vec{x}}, \quad v_{k}^{*}(\vec{x})=\frac{1}{\sqrt{V}} e^{-i \vec{k} \cdot \vec{x}}$, are normalized in a finite volume $V$. Separating the real and imaginary parts of $\phi_{k}(t)$ as

$\phi_{k}(t)=\frac{1}{\sqrt{2}}\left(\phi_{k}^{1}+i \phi_{k}^{2}\right)$,

one obtains for each mode $k$ and $j$ the action

$S=\frac{1}{2} \sum_{k} \sum_{j=1,2} \int e^{3 H_{0} \tau} \mathrm{d} \tau\left(\dot{\phi}_{k}^{j 2}-\omega_{k}^{2} \phi_{k}^{j 2}\right)$,

where $\omega_{k}$ is a frequency given by [18-20]

$\omega_{k}^{2}(t)=m^{2}+k^{2} e^{-2 H_{0} t}$.

Henceforth, for simplicity we will denote collectively $k, j$ by $k$ since from Eq. (17) we see that the modes $k$ and $j$ are independent and may be considered separately $[18,19]$. Then, from Eq. (17) one obtains for each mode $k$ the following Hamiltonian:

$H_{k}=\frac{\pi_{k}^{2}}{2 a^{3}(t)}+\frac{1}{2} a^{3}(t) \omega_{k}^{2}(t) \phi_{k}^{2}, \quad a(t)=e^{H_{0} t}$,

where

$\pi_{k}=a^{3}(t) \dot{\phi}_{k}$,

is the generalized conjugate momentum. By making use of the Hamiltonian (19) one readily finds that the classical equation of the motion for the mode $\phi_{k}$ can be written as

$\ddot{\phi}_{k}+3 H_{0} \dot{\phi}_{k}+\omega_{k}^{2} \phi_{k}=0$.

The solution of this equation is given by [18-20]

$\phi_{k}(t)=\left(\frac{\pi}{4 H_{0}}\right)^{1 / 2} e^{-(3 / 2) H_{0} t} H_{v}^{(2)}(z)$,

where $H_{v}^{(2)}$ is the Hankel function of the second kind with

$v=\left(\frac{9}{4}-\frac{m^{2}}{H_{0}^{2}}\right)^{1 / 2} \quad, \quad z=\frac{k}{H_{0}} e^{-H_{0} t}$.

Then, as the mode and amplitude functions are completely specified, we can write the scalar field $\Phi(\vec{x}, t)$ [see Eqs. (14), (15)] as

$\Phi(\vec{x}, t)=\frac{1}{\sqrt{V}} \sum_{k} e^{i \vec{k}_{l} \cdot \vec{x}} \phi_{k}(t)$,

with $\phi_{k}$ given by Eq. (22). From Eqs. (22) and (24) we see that the scalar field also decay with time but now this attenuation is in proportion to $\exp \left(-3 H_{0} t / 2\right)$. 
At this point, we observe that, from the above results, the mathematical formalism to describe the classical light propagating through a linear dielectric medium with a timevarying electric permittivity increasing exponentially and the classical propagation of a scalar field propagating in a de Sitter spacetime is identical. In both cases the vector potential $\vec{A}(\vec{r}, t)$ and the scalar field $\Phi(\vec{x}, t)$ are written in terms of mode and amplitude functions [see Eqs. (3) and (14)], the equations of motion for the amplitude functions [see Eqs. (8) and (21)] are governed by similar Hamiltonians [see Eqs. (7) and (19)] and the mode functions are written in terms of plane waves [see Eqs. (10) and (24)]. This analogy can be realized by making the following correspondence: $\vec{r} \leftrightarrow \vec{x}$, $\varepsilon(t) \leftrightarrow a(t), q_{l} \leftrightarrow \phi_{k}$ and $\Omega_{l} \leftrightarrow \omega_{k}$. We also note that the correspondence $\varepsilon(t) \leftrightarrow a(t)$ and the attenuation of $\Phi(\vec{x}, t)$ [see Eqs. (22) and (24)], show that the de Sitter background plays the role of the time-dependent dielectric medium in the propagation of the scalar field (see comment at the end of the first paragraph of Sect. 2). However, the equivalence of the behavior of these two systems is not complete. The dispersion relations [see Eqs. (6) and (18)], which are inherent to each physical system, differ and as a consequence the behavior of each amplitude mode is different. Therefore, this difference in the dispersion relation leads to a different dynamic evolution for these two systems. We also observe that even in the massless limit for the scalar field, that is, $m=0$, the dispersion relations are different. In fact, the modified frequency is proportional to $1 / e^{H_{0} t}$ in the de Sitter spacetime whereas it is proportional to $1 / \sqrt{e^{\eta t}}$ in the dielectric problem and consequently the behavior of each mode is also different.

\section{Quantum scalar fields in a de Sitter spacetime}

In this section our objective is to analyze the quantum propagation of a scalar field in a de Sitter spacetime. To this end, it is necessary to solve the time-dependent Schrödinger equation associated with the Hamiltonian (19). For this problem, this equation can written as

$H_{k}|\Psi, t\rangle=i \hbar \frac{\partial}{\partial t}|\Psi, t\rangle$

where the amplitude mode $\phi_{k}(t)$ and the generalized momentum $\pi_{k}$ are now canonically conjugate operators satisfying the relation $\left[\phi_{k^{\prime}}, \pi_{k}\right]=i \hbar \delta_{k^{\prime} k}$ with $\pi_{k}=-i \hbar \partial / \partial \phi_{k}$. The solutions of this equation can be readily derived with the aid of the dynamical invariant method introduced by Lewis and Riesenfeld [25]. Following the steps of Ref. [25] we must look for a nontrivial Hermitian operator $I_{k}(t)$ which fulfills the equation

$\frac{\mathrm{d} I_{k}}{\mathrm{~d} t}=\frac{1}{i \hbar}\left[I_{k}, H_{k}\right]+\frac{\partial I_{k}}{\partial t}=0$.
Then the solutions of the Schrödinger equation (25) can be written in terms of orthonormalized eigenstates $\left|\phi_{n_{k}}, t\right\rangle$ of $I_{k}(t)$

$\left.\left.I_{k}(t) \mid \phi_{n_{k}}, t\right)\right\rangle=\lambda_{n_{k}}\left|\phi_{n_{k}}, t\right\rangle$,

and the phase functions $\beta_{n_{k}}(t)$ as

$\left|\psi_{n_{k}}, t\right\rangle=e^{i \beta_{n_{k}}(t)}\left|\phi_{n_{k}}, t\right\rangle$.

The $\lambda_{n_{k}}$ are time-independent eigenvalues and the phase functions $\beta_{n_{k}}(t)$ are obtained from the equation

$\hbar \frac{\mathrm{d} \beta_{n_{k}}(t)}{\mathrm{d} t}=\left\langle\phi_{n_{k}}, t\left|i \hbar \frac{\partial}{\partial t}-H_{k}(t)\right| \phi_{n_{k}}, t\right\rangle$,

with the orthonormality condition $\left\langle\phi_{n_{k}^{\prime}}, t \mid \phi_{n_{k}}, t\right\rangle=\delta_{n_{k}^{\prime} n_{k}}$.

Now, it is well known that a quadratic invariant for the Hamiltonian (19) is given by $[4,12]$

$I_{k}(t)=\frac{1}{2}\left[\left(\frac{\phi_{k}}{\rho_{k}}\right)^{2}+\left[\rho_{k} \pi_{k}-a^{3} \dot{\rho}_{k} \phi_{k}\right]^{2}\right]$,

where $\rho_{k}(t)$ is a time-dependent real function satisfying the Milne-Pinney equation [26,27]

$\ddot{\rho}_{k}(t)+3 H_{0} \dot{\rho}_{k}(t)+w_{k}^{2} \rho_{k}=\frac{e^{-6 H_{0} t}}{\rho_{k}^{3}}$.

The solutions of this equation can be written as $[18,19]$

$$
\begin{aligned}
\rho_{k}(t)= & a^{-3 / 2}\left[A_{0} J_{v}^{2}(z)+B_{0} N_{v}^{2}(z)\right. \\
& \left.+2\left(A_{0} B_{0}-\frac{\pi^{2}}{4 H_{0}^{2}}\right)^{1 / 2} J_{v}(z) N_{v}(z)\right]^{1 / 2},
\end{aligned}
$$

where $J_{v}$ and $N_{v}$ are Bessel functions and $A_{0}$ and $B_{0}$ are real constants.

Our next step is to find the eigenstates of the invariant $I_{k}(t)$. To do this, let us introduce annihilation- and creationtype operators $b_{k}(t)$ and $b_{k}^{\dagger}(t)$ defined by $[4,25,28]$

$$
\begin{aligned}
& b_{k}(t)=\left(\frac{1}{2 \hbar}\right)^{1 / 2}\left[\frac{\phi_{k}}{\rho_{k}}+i\left(\rho_{k} \pi_{k}-a^{3} \dot{\rho}_{k} \phi_{k}\right)\right], \\
& b_{k}^{\dagger}(t)=\left(\frac{1}{2 \hbar}\right)^{1 / 2}\left[\frac{\phi_{k}}{\rho_{k}}-i\left(\rho_{k} \pi_{k}-a^{3} \dot{\rho}_{k} \phi_{k}\right)\right],
\end{aligned}
$$

with

$$
\left[b_{k}(t), b_{k}^{\dagger}(t)\right]=1 \text {. }
$$

In terms of these operators, the invariant (21) can be factored as

$$
I_{k}(t)=\hbar\left[b_{k}^{\dagger}(t) b_{k}(t)+\frac{1}{2}\right] .
$$


By making use of (35) and (36) the eigenvalue equation for $I_{k}(t)$ (see Eq. (27)) can also be solved exactly, just as for harmonic oscillator in the time-independent case by using the Fock states $\left|n_{k}, t\right\rangle$. Then, defining the Hermitian number operator by $N_{k}=b_{k}^{\dagger} b_{k}$ so that $N_{k}\left|n_{k}, t\right\rangle=n_{k}\left|n_{k}, t\right\rangle$, we get

$$
\begin{aligned}
& I_{k}(t)=\hbar\left(N_{k}+\frac{1}{2}\right) . \\
& I_{k}(t)\left|n_{k}, t\right\rangle=\hbar\left(n_{k}+\frac{1}{2}\right)\left|n_{k}, t\right\rangle, \\
& b_{k}(t)\left|n_{k}, t\right\rangle=n_{k}^{1 / 2}\left|n_{k}-1, t\right\rangle, \\
& b_{k}^{\dagger}\left|n_{k}, t\right\rangle=\left(n_{k}+1\right)^{1 / 2}\left|n_{k}+1, t\right\rangle .
\end{aligned}
$$

After making the change $\left|\phi_{n_{k}}, t\right\rangle \rightarrow\left|n_{k}, t\right\rangle$ the phase function is calculated from (29) which yields $[4,12,25]$

$\beta_{n_{k}}(t)=-\left(n_{k}+\frac{1}{2}\right) \int_{0}^{t} \frac{1}{a^{3}(\tau) \rho_{k}^{2}(\tau)} \mathrm{d} \tau$.

Therefore, the solutions of the Schrödinger equation (25) can be written as

$\left|\psi_{n_{k}}, t\right\rangle=e^{i \beta_{n_{k}}(t)}\left|n_{k}, t\right\rangle$,

with $\beta_{n_{k}}(t)$ given by (41). The general solution to the Schrödinger equation (25) can be written as $|\Psi, t\rangle=$ $\sum_{n_{k}} c_{n_{k}}\left|\psi_{n_{k}}, t\right\rangle$, where the coefficients $c_{n_{k}}$ are constants.

Now, from (33) and (34) we obtain

$\phi_{k}(t)=\left(\frac{\hbar}{2}\right)^{1 / 2} \rho_{k}\left[b_{k}(t)+b_{k}^{\dagger}(t)\right]$.

Thus, using Eqs. (14), (15) and (43) we can write the scalar field $\Phi(\vec{x}, t)$ in the form $[1,7,19]$

$\Phi(\vec{x}, t)=\frac{1}{\sqrt{V}} \sum_{k} \rho_{k}(t)\left[e^{i \vec{k}_{k} \cdot \vec{x}} b_{k}(t)+e^{-i \vec{k}_{k} \cdot \vec{x}} b_{k}^{\dagger}(t)\right]$,

where $\rho_{k}(t)$ is given by Eq. (32). Therefore, the above expression represents the scalar field in a de Sitter spacetime.

In the following, we calculate the expectation values of $\phi_{k}(t), \pi_{k}(t)$, their variances and the respective uncertainty principle in the state $\left|n_{k}, t\right\rangle$. By using Eqs. (39) and (40) we find that

$$
\begin{aligned}
& \left\langle I_{k}\right\rangle=\hbar\left(n_{k}+\frac{1}{2}\right), \\
& \left\langle\phi_{k}\right\rangle=\left\langle\pi_{k}\right\rangle=0, \\
& \left\langle\phi_{k}^{2}\right\rangle=\hbar \rho_{k}^{2}\left(n_{k}+\frac{1}{2}\right), \\
& \left\langle\pi_{k}^{2}\right\rangle=\hbar\left[\frac{1}{\rho_{k}^{2}}+\left(a^{3} \dot{\rho}_{k}\right)^{2}\right]\left(n_{k}+\frac{1}{2}\right) .
\end{aligned}
$$

The quantum variances are expressed as

$$
\begin{aligned}
& \left(\Delta \phi_{k}\right)^{2}=\left\langle\phi_{k}^{2}\right\rangle-\left\langle\phi_{k}\right\rangle^{2}=\hbar \rho_{k}^{2}\left(n_{k}+\frac{1}{2}\right), \\
& \left(\Delta \pi_{k}\right)^{2}=\left\langle\pi_{k}^{2}\right\rangle-\left\langle\pi_{k}\right\rangle^{2}=\hbar\left[\frac{1}{\rho_{k}^{2}}+\left(a^{3} \dot{\rho}_{k}\right)^{2}\right]\left(n_{k}+\frac{1}{2}\right),
\end{aligned}
$$

so that the uncertainty principle can be written as

$\left(\Delta \phi_{k}\right)\left(\Delta \pi_{k}\right)=\hbar\left[1+\left(a^{3} \rho_{k} \dot{\rho}_{k}\right)^{2}\right]^{1 / 2}\left(n_{k}+\frac{1}{2}\right)$.

Finally, it is worth remarking that the quantum behavior of the propagation of light in a dielectric medium with a timevarying electric permittivity that increases exponentially can be carried out following the same procedure developed in this section, since as we have demonstrated in Sects. 2 and 3 , the mathematical framework to describe both systems is identical.

\section{Coherent states for the quantum scalar field}

We now use the results of the previous section to construct coherent states for the system described by the Hamiltonian (25). In Ref. [29] Hartley and Ray constructed coherent states for a time-dependent mechanical harmonic oscillator. Thus, following the same steps as these authors we find that the coherent states for the quantum system described by the Hamiltonian (19) are given by

$$
\left|\alpha_{k}, t\right\rangle=\exp \left(-\frac{\left|\alpha_{k}\right|^{2}}{2}\right) \sum_{n_{k}} \frac{\left(\alpha_{k}\right)^{n_{k}}}{\left(n_{k} !\right)^{1 / 2}} \exp \left[i \beta_{n_{k}}(t)\right]\left|n_{k}, t\right\rangle
$$

where $\alpha_{k}$ is an arbitrary complex number. Next, we examine some properties of these states. They are eigenstates of the annihilation operator $b_{k}(t)$

$b_{k}\left|\alpha_{k}, t\right\rangle=\alpha_{k}(t)\left|\alpha_{k}, t\right\rangle$,

with the eigenvalue $\alpha_{k}(t)$ being given by

$\alpha_{k}(t)=\alpha_{k} e^{2 i \beta_{0} t}$,

where

$\beta_{0}(t)=-\frac{1}{2} \int_{0}^{t} \frac{1}{a^{3}(\tau) \rho_{k}^{2}(\tau)} \mathrm{d} \tau$. 
On the other hand, the calculation of the quantum variances of $\phi_{k}$ and $\pi_{k}$ in the coherent state $\left|\alpha_{k}, t\right\rangle$ yields

$$
\begin{aligned}
\left\langle\Delta \phi_{k}\right\rangle^{2} & =\left\langle\phi_{k}^{2}\right\rangle-\left\langle\phi_{k}\right\rangle^{2}=\frac{\hbar}{2} \rho_{k}^{2}, \\
\left\langle\Delta \pi_{k}\right\rangle^{2}=\left\langle\pi_{k}^{2}\right\rangle-\left\langle\pi_{k}\right\rangle^{2} & =\frac{\hbar}{2}\left[\frac{1}{\rho_{k}^{2}}+\left(a^{3} \dot{\rho}_{k}\right)^{2}\right],
\end{aligned}
$$

so that the uncertainty principle is

$\left(\Delta \phi_{k}\right)\left(\Delta \pi_{k}\right)=\frac{\hbar}{2}\left[1+\left(a^{3} \rho_{k} \dot{\rho_{k}}\right)^{2}\right]^{1 / 2}$.

By comparing Eqs. (51) and (58) we see that the uncertainty principle in the coherent states is exactly the same as the minimum value of that in the Fock states $\left|n_{k}, t\right\rangle$. It may be helpful in this point to observe that the coherent states $\left|\alpha_{k}, t\right\rangle$ are not states of minimum uncertainty. This occurs because the states $\left|\alpha_{k}, t\right\rangle$ indeed correspond to the squeezed states [3034]. Finally, it is worth noticing that following the same steps as in this section one can construct coherent states for the quantized light propagating in a dielectric medium with a time-varying permittivity increasing exponentially.

\section{Summary}

In the present work, we have analyzed the behavior of light propagating in a dielectric medium with a time-varying electric permittivity increasing exponentially and the propagation of a scalar field in a de Sitter spacetime. We have shown that the mathematical framework to study the behavior of these two different physical systems is identical, both classically and quantum mechanically, and can be described by similar Hamiltonians. Moreover, we have noted that de Sitter background plays the role of the time-dependent dielectric medium in the propagation of the scalar field. On the other hand, we have seen that the dispersion relations of these systems, which are inherent to each physical system, are different and consequently lead to a different dynamic evolution for both systems. Further, using the invariant method, quadratic invariants, appropriate annihilation- and creationtype operators and Fock states we have easily solved the timedependent Schrödinger for the Hamiltonian that describe the scalar field in a de Sitter spacetime and have written its solutions in terms of solutions of the non-linear Milne-Pinney equation. We have used these solutions to construct coherent states for the quantized scalar field and have calculated the quantum variances of the amplitude mode $\phi_{k}(t)$ and generalized momentum $\pi_{k}(t)$ as well as the uncertainty principle for each mode of the quantized scalar field in both states, namely, Fock and coherent states. We also have seen that the uncertainty product in the coherent states is equal to the minimum value of that of the Fock states. Finally, we expect that the present work can stimulate other theoretical physicists to investigate other different physical systems which can be described by the same mathematical formalism.

Acknowledgements The authors K. Bakke and B. F. Ramos would like to thank $\mathrm{CNPq}$ for partial financial support.

Author contributions The authors contributed equally to the paper.

Data Availability Statement This manuscript has no associated data or data will not be deposited. [Authors' comment: The authors have not deposited any data. However, any question about the article may be asked to the authors via email.]

Open Access This article is licensed under a Creative Commons Attribution 4.0 International License, which permits use, sharing, adaptation, distribution and reproduction in any medium or format, as long as you give appropriate credit to the original author(s) and the source, provide a link to the Creative Commons licence, and indicate if changes were made. The images or other third party material in this article are included in the article's Creative Commons licence, unless indicated otherwise in a credit line to the material. If material is not included in the article's Creative Commons licence and your intended use is not permitted by statutory regulation or exceeds the permitted use, you will need to obtain permission directly from the copyright holder. To view a copy of this licence, visit http://creativecomm ons.org/licenses/by/4.0/.

Funded by $\mathrm{SCOAP}^{3}$.

\section{References}

1. W.H. Louisell, Quantum Statistical Properties of Radiation (Wiley, New York, 1973)

2. J.R. Choi, S. Lakehal, M. Maamache, S. Menouar, Progr. Electromagn. Res. Lett. 44, 71 (2014)

3. R.J. Glauber, Quantum Theory of Particle by Oscillating Fields (Lyman Laboratory of Physics, Havard University, Cambridge, 1991)

4. I.A. Pedrosa, Phys. Rev. A 83, 032108 (2011)

5. R. Matloob, R. Loudon, S.M. Barnett, J. Jeffers, Phys. Rev. A 52, 4823 (1995)

6. R.J. Glauber, M. Lewenstein, Phys. Rev. A 467, 43 (1991)

7. J.R. Choi, J. Opt. B 5, 409 (2003)

8. J.R. Choi, Int. J. Theor. Phys. 43, 2113 (2004)

9. J.R. Choi, J. Phys. B 39, 669 (2006)

10. J.R. Choi, Phys. Rev. A 82, 055803 (2010)

11. H. Lakehal, M. Maamache, J. Choi, Sci. Rep. 6, 19860 (2016)

12. A.L. de Lima, A. Rosas, I.A. Pedrosa, J. Phys. B 41, 115503 (2008)

13. I.A. Pedrosa, A. Rosas, Phys. Rev Lett. 103, 010402 (2009)

14. I.A. Pedrosa, C. Furtado, A. Rosas, EPL 94, 30002 (2011)

15. M.M.M. Carvalho, C. Furtado, I.A. Pedrosa, Phys. Rev. D 70, $123523(2004)$

16. G. Alencar, I. Guedes, R.R. Landim, R.N.C. Filho, EPL 98, 11001 (2012)

17. G. Alencar, I. Guedes, R.R. Landim, R.N. Costa Filho, Int. J. Mod. Phys. A 27, 1250177 (2012)

18. C. Bertoni, F. Finelli, G. Venturi, Phys. Lett. A 237, 331 (1998)

19. F. Finelli, A. Gruppuso, G. Venturi, Class. Quantum Gravity 16, $3923(1999)$

20. C.E.F. Lopes, I.A. Pedrosa, C. Furtado, A.M.M. Carvalho, J. Math. Phys. 50, 083511 (2009)

21. K. Bakke, I.A. Pedrosa, C. Furtado, J. Math. Phys. 50, 113521 (2009) 
22. P.M. Alsing, J.P. Dowling, G.J. Milburn, Phys. Rev. Lett. 94, 220401 (2005)

23. N.C. Mennicucci, G.J. Milburn, Phys. Rev. A 76, 052105 (2007)

24. L.D. Landau, E.M. Lifshitz, The Classical Theory of Fields: Fourth Revised English Edition (Course of Theoretical Physics Series), vol. 2, Chapter 10, $\$ 90(1987)$

25. H.R. Lewis Jr., W.B. Riesenfeld, J. Math. Phys. (N.Y.) 10, 1458 (1969)

26. W.E. Milne, Phys. Rev. 35, 863 (1930)

27. W.K. Schief, Appl. Math. Lett. 10, 13 (1997)
28. I.A. Pedrosa, Phys. Rev. A 55, 3219 (1997)

29. J.G. Hartley, J.R. Ray, Phys. Rev. D 25, 382 (1982)

30. D. Stoler, Phys. Rev. D 1, 3217 (1970)

31. H.P. Yuen, Phys. Rev. A 13, 2226 (1976)

32. I.A. Pedrosa, Hadron. J. 9, 173 (1986)

33. D.F. Walls, Nature 306, 141 (1983)

34. I.A. Pedrosa, V.B. Bezerra, Mod. Phys. Lett. A 12, 1111 (1997) 